\title{
Aripiprazol Versus Quetiapine in Treatment of Non-affective Acute Psychosis: A Double-Blind, Randomized - Controlled Clinical Trial
}

\author{
S. Mohamad Moosavi ${ }^{1}$, Mahshid Ahmadi ${ }^{2}$, Mani B. Monajemi ${ }^{3}$ * \\ ${ }^{1}$ Department of Psychiatry, Mazandaran University of Medical Sciences, Sari, Iran \\ ${ }^{2}$ Department of Social Medicine, Mazadaran University of Medical Sciences, Sari, Iran \\ ${ }^{3}$ School of Psychology and Mental Health Sciences, University of Tehran, Tehran, Iran
}

\section{Email address:}

mani.b.monajemi@warwickgrad.net (M. B. Monajemi), mani.b.monajemi@ut.ac.ir (M. B. Monajemi)

${ }^{*}$ Corresponding author

\section{To cite this article:}

S. Mohamad Moosavi, Mahshid Ahmadi, Mani B. Monajemi. Aripiprazol Versus Quetiapine in Treatment of Non-affective Acute Psychosis: A Double-Blind, Randomized - Controlled Clinical Trial. American Journal of Psychiatry and Neuroscience. Vol. 5, No. 1, 2017, pp. 6-9. doi: 10.11648/j.ajpn.20170501.12

Received: July 24, 2016; Accepted: December 14, 2016; Published: February 21, 2017

\begin{abstract}
Objective: The primary objective of this study was to assess the efficacy of Quetiapine versus Aripiprazol in treating non-affective acute psychosis. Methods: This study is double blind, randomized-controlled clinical trial (RCT). A total number of 90 hospitalized patients with the diagnosis of acute psychosis were selected. The patients were treated with Quetiapine (mean: $500 \mathrm{mg} /$ day) or Aripiprazol (mean: $20 \mathrm{mg} /$ day), in a 4 weeks period. The positive and negative symptoms scale (PANSS) and clinical global impression severity scale (CGIS-s) were used in the first and the last day of trial. Results: This study reveals that both of these drugs can substantially reduce the severity of symptoms in acute psychosis. Aripiprazol reduced positive symptoms and general symptoms scores and total scores more than Quetiapine. Differences in reducing negative scores were not significant and both drugs had the same therapeutic effect. In this sense, there was no difference in reducing negative symptoms in acute psychosis between Aripiprazol and Quetiapine. Conclusion: According to some differences with similar studies, study on larger sample pool and in longer period in future researches can be advised.
\end{abstract}

Keywords: Antipsychotics, Aripiprazol, Quetiapine, Non-affective Acute Psychosis

\section{Introduction}

Psychosis is a mental state spectrum accompanied by impairment of thoughts, emotional responses and interactions. This group of disorders often interfere the ability of reality testing. Classic features of psychosis include hallucination and delusion [1]. Suitable and impeccable timing in treatment is associated with enhanced clinical response and hinders severity of this malady [2]. A revolution conceived by chlorpromazine for treating psychosis in 1952 [3]. Second generation antipsychotics (SGAs) have been available since 1990. These drugs are serotonin-dopamine receptors antagonist. The first drug of this group was Clozapine. Resperidone, Olanzapine, Quetiapine, Ziprasidone and Aripprazole were produced later [4]. Second Generation
Antipsychotics may considered more effective comparing to First Generation Antipsychotics (FGAs) in controlling positive and negatives symptoms of psychosis. Admittedly, they may have less detrimental effects and using these drugs may decrease the number of drug resistant patients. Furthermore, they may decrease the relapse rate and longterm hospitalization [4]. The evidence regarding best option among antipsychotics in treatment of psychotic disorders is limited. The objective of this study was to compare the efficacy of Quetiapine versus Aripiprazole in treatment of non-affective psychosis. Hopefully, the results would help clinicians to implement proper therapeutic approach. Very few RCTs have compared Quetiapine with Aripiprazole 
among adults in Iran. Regarding uprising trend in production and using of SGAs in the recent years and contradictory results of different studies, we decided to compare the efficacy of these two drugs in treatment of acute phase of psychosis.

\section{Materials and Methods}

This study is double blind, randomized-controlled clinical trial (RCT). Patients in a psychiatric Hospital (Sari, Iran) $(\mathrm{n}=100)$ were randomly assigned to 2 groups (Aripiprazole, Quetiapine) for 4 weeks intervention period with Quetiapine and Aripiprazole. We selected Quetiapine and Aripiprazole since these drugs are frequently used in psychiatric settings, and because it is unclear whether their different receptor binding profiles can be related to difference clinical outcomes.

Inclusion criteria: 1- adults aged 18-50 years, both sexes (matched for ages \& sexes); 2- inpatients in psychiatric Hospital; 3- meeting the criteria for DSM-5 psychosis diagnoses (non-organic, non-drug-induced): Schizophrenia, delusional disorders, shared delusional disorders, Schizoaffective disorders; 4-clinical indication for antipsychotic treatment; 5- presence of psychotic symptoms scoring $\geq 4$ on at least one of the following PANSS items: delusions, conceptual disorganization, hallucinations, grandiosity, suspiciousness/persecution, or unusual thought content; as well as a total PANSS score $>60$ points; 6antipsychotic-naïve or limited exposure (no use antipsychotics orally 6 weeks prior to evaluation and regarding long acting antipsychotics this period defined as 8 weeks) 7- written informed consent by caretakers (Regarding the type of the illness and its acute phase, written consent by patients was useless legally and morally. Instead, the process of the study explained for the parents or caretakers and consent by them).

Exclusion criteria: 1- drug-induced or organic psychosis; 2- severe chronic somatic and neurological illness or a history of severe head trauma (based on physical and neurological examinations at the first day) and abnormal laboratory tests (CBC, FBS, BUN, Creatinine, ALT, AST, Alkaline Phosphatase, U/A and also ECG); 3- pregnancy or lactation; 4- substance dependence ( based on DSM-5 and laboratory tests) within the last year; 5- Consuming other antipsychotic drugs even in PRN situation, 6- Electro Convulsive therapy (ECT) based on attending physician order; 7-Prescribing any drugs except Aripiprazol and Quetiapine during hospitalization. This study was conducted in accordance with the declaration of Helsinki \& good clinical practice according to international conference on harmonization guidelines.

The interventions are blinded to participants, caregivers, statisticians, and conclusion drawers.

The maximum doses are Quetiapine $800 \mathrm{mg} /$ day vs Aripiprazole $30 \mathrm{mg} / \mathrm{day}$. However, if needed, dosing is flexible (Quetiapine: 100-800 mg/day; Aripiprazole 5-30 $\mathrm{mg}$ /day). Beneficial and harmful effects were assessed at two time points during the intervention period (weeks 2, 4).

G-Power software was used and considering potency of 80 , sample size was 90 . Regarding to probability of exclusion for any reason and due to unpredicted problems during the study, 141 patients had been chosen for study. The patients were divided in two groups using random numbers nature. Excel software and Rand between function were also used. The primary outcome is positive symptoms measured on the PANSS scale, Clinical interview for all patients by a psychiatrist and also CGI scale [5] was used in order to assess the severity of the illness. Positive and negative symptoms scale (PANSS) was used to specify the severity of negative and positive and general psychiatric symptoms. The latter scales include 7 negative symptoms, 7 positive symptoms and 14 general psychopathologic symptoms. Each symptom scored between 0-7 based on severity. Clinicians widely in researches with respect to antipsychotic drugs use this scale. Ghamari and colleagues confirmed validity and reliability of this Scale in Iranian population pool [6].

Each group of patients, were under Aripiprazol or Quetiapine treatment. In this study first drug (Aripiprazol) was chosen from Jam Pharmaceutical Company and the second (Quetiapine) was produced by Jam Co. Initial dosage of neuroleptic drugs in common situation based on researches could be $2.5 \mathrm{mg}$ for Aripiprazol and $50 \mathrm{mg}$ for Quetiapine Per-day [7]. The initial dosage was $5 \mathrm{mg}$ for Aripiprazol and $100 \mathrm{mg}$ for Quetiapine group due the acute situation of the patients in this study. Maximum daily dosage was $30 \mathrm{mg}$ for Aripiprazol and $800 \mathrm{mg}$ for Quetiapine [7]. At the end of week 4, mean dosages of drug for each patient were measured. Based on statistical survey prior to this study, the average duration of hospitalization period was 27 days, so and we measured the dosage of drugs after week 4.

At the end of the study, participants were assessed again by PANSS and CGIS scales. Furthermore Electrocardiography (ECG), blood pressure, pulse, body mass index, abdominal circumference, laboratory test results were assessed.

Data were analyzed by SPSS software version 19. In order to analyze data, for quantitative variables such as age and PANSS, descriptive statistics method such as Mean \pm standard deviation (SD) was used and for qualitative variables, distribution frequency chart was used. In order to compare the effectiveness of the drugs we used Analyze of Variance with repetitive measurement of Chi-square and one-way Analyze of Variance with Bonferoni post-test. If groups were not the same in terms of cofounding variables, Generalized Estimated Equations (GEE) method was used. $P$ value less than 0.05 were defined as significant in this study.

\section{Results}

One hundred forty one patients were included in this study. 7 patients discharged before 4 weeks were excluded from our study. 15 patients needed ECT according to comments of 
attending physician; they were excluded from the study too. 19 patients have been under with PRN. Finally, study was conducted on 100 patients.

Patients were included 33 women and 67 men. 16 women were in Aripiprazol group and 17 were in Quetiapine group. 32 in first group and 35 in second groups were male. The Mean \pm SD age of patients whom underwent Aropiprazol was $36.5 \pm 8.3$ versus $34.2 \pm 9.3$ in Quetiapine group. In this relation, there was no significant difference between the age of study arms $(\mathrm{p}=0.478) .32$ patients in Aripiprazol group and 35 in Quetiapine group were male while 16 in Aripiprazol group and 17 in Quetiapine group were female. There was no significant change in studied patients gender $(\mathrm{p}=0.791)$ (Table 1).
Table 1. Age \& Sex Distribution in Aripiprazol and Quetiapine Groups.

\begin{tabular}{lllll}
\hline \multirow{2}{*}{ Characteristics } & \multicolumn{2}{l}{ Treatment } & \multirow{2}{*}{ P-Value } \\
\cline { 2 - 4 } & Aripiprazol & Quetiapine & \\
\hline \multirow{2}{*}{ Age } & 36.5 & 34.2 & 34.2 & 0.478 \\
& 11.6 & 12.3 & 12.3 & \\
\multirow{2}{*}{ Sex } & 32 & 35 & 35 & 0.791 \\
& 16 & 17 & 17 & \\
\hline
\end{tabular}

In this Study, mean dosage for Risperidone was $14.6 \mathrm{mg}$ and mean dosage for Quetiapine was $509 \mathrm{mg}$. $100 \mathrm{mg}$ chlorpromazine is equivalent to $5 \mathrm{mg}$ Aripiprazol and $75 \mathrm{mg}$ Quetiapine. Hence, $436 \mathrm{mg}$ for Aripiprazol with equivalent dose of Chlorpromazine and $664 \mathrm{mg}$ for Quetiapine was specified. Above means we used higher dose of Aripiprazol in this study.

Table 2. Comparing PANSS AND CGI-S, Baseline and Week 4.

\begin{tabular}{|c|c|c|c|c|c|}
\hline \multirow[b]{2}{*}{ Treatment } & & \multicolumn{2}{|l|}{ Baseline } & \multicolumn{2}{|c|}{ Diff. After 4 weeks } \\
\hline & & Mean & P-value & Mean & P-value \\
\hline \multirow{2}{*}{ CGI-s } & Aripiprazol & $5.71 \pm 0.48$ & \multirow{2}{*}{0.237} & $1.36 \pm 0.58$ & \multirow{2}{*}{0.001} \\
\hline & Quetiapine & $5.81 \pm 0.43$ & & $1.44 \pm 0.73$ & \\
\hline \multirow{2}{*}{ Positive symptoms. } & Aripiprazol & $23.26 \pm 1.88$ & \multirow[t]{2}{*}{0.834} & $14.49 \pm 2.47$ & \multirow{2}{*}{0.045} \\
\hline & Quetiapine & $24.29 \pm 1.78$ & & $11.44 \pm 2.51$ & \\
\hline Negative symptoms. & Aripiprazol & $18.02 \pm 3.12$ & 0.579 & $3.56 \pm 1.39$ & 0.286 \\
\hline \multirow{2}{*}{ General symptoms. } & Aripiprazol & $48.24 \pm 4.1$ & \multirow{2}{*}{0.185} & $18.24 \pm 1.58$ & \multirow{2}{*}{0.001} \\
\hline & Quetiapine & $47.15 \pm 3.92$ & & $14.4 \pm 1.92$ & \\
\hline \multirow{2}{*}{ Total } & Aripiprazol & $93.43 \pm 5.17$ & \multirow{2}{*}{0.611} & $22.2 \pm 5.18$ & \multirow{2}{*}{0.031} \\
\hline & Quetiapine & $93.16 \pm 6.27$ & & $19.1 \pm 4.66$ & \\
\hline
\end{tabular}

Two groups had no statistically meaningful difference in relation with CGI-s scores from start point to the end of this study $(p<0.001)$ but effectiveness of both drugs in reducing CGI-s was meaningful. In PANSS sub-scores (General/Positive/Negative), there were no meaningful difference at baseline. At the end of week 4, no statistically difference in reducing positive and negative symptoms observed between Aripiprazol and Quetiapine groups whereas differences between two groups were significant regarding positive symptoms and general symptoms (Table 2). It means Aripiprazol was more effective than Quetiapine in reducing positive and general symptom scores of PANSS in acute phase of psychosis. Furthermore, the difference between two groups in total scores was statistically meaningful (Table 2) and this confirms superior effectiveness of Aripiprazol over Quetiapine in treating acute phase of psychosis.

\section{Discussion}

In this double blind randomized controlled study, we examined the efficacy of Aripiprazol versus Quetiapine in treatment of acute psychosis. This study revealed that both of these drugs are efficient in reducing severity of acute psychosis. Aripiprazole reduced PANSS in positive symptoms and general symptoms scores and total scores more than Quetiapine in acute psychosis. Differences in reducing negative scores were not significant and both drugs had the same therapeutic effects. It means there was no difference in reducing negative symptoms in acute psychosis between Aripiprazole and Quetiapine.

Misso and colleagues (2013) reported Aripiprazole with potentiate treatment in nonresponders to Quetiapine can provide relevant information on the effectiveness of Aripiprazol in clinical practice [8]. Different from current study, their study conducted among patients with bipolar disorder. In another study conducted by Jin and colleagues (2013) lack of significant difference in improvement in psychopathology for all atypical antipsychotics have been indicated [9]. No difference was found in medication compliance in patients with schizophrenia or bipolar disorder, who initiate treatment with Aripiprazole, Quetiapine, or Ziprasidone [10]. Rates of efficacy failure were similar between second-generation antipsychotics groups, but switching to Aripiprazole was associated with a higher rate of treatment discontinuation according to Stroup's study [11] No significant differences were detected in risk of outcome across 5 commonly prescribed second-generation antipsychotic medications. [12] There are differences in the results of studies as mentioned above.

\section{Limitation}

1 The trial was with patients undergoing acute treatment (phase I). Potentiation (phase II) and maintenance (phase III) (long-term treatment) is an issue of great 
importance and should be evaluated further through more in-depth studies given that psychosis is a chronic disease.

2 It is advisable to conduct a study in larger sample pool and in longer period in future researches.

\section{Authors' Contributions}

SMM and MBM conceived and designed the evaluation. MBM performed the statistical analysis. MA drafted the manuscript. All authors read and approved the manuscript.

\section{Acknowledgement}

We are very grateful to all patient participants at Zare Psychiatric Hospital and their families for their cooperation in this study.

\section{References}

[1] J. Sadock B: Signs and symptoms in psychiatry. Kaplan \&Sadock's Comprehensive Textbook of Psychiatry 9th Edition 927.

[2] Kane JM, Stroup TS, Marder SR: Schizophrenia: Pharmacological Treatment. Kaplan \& Sadock's Comprehensive Textbook of Psychiatry 9th Edition: 1547.

[3] Kane JM, Stroup TS, Marder SR: Schizophrenia: Pharmacological Treatment. Kaplan \& Sadock's Comprehensive Textbook of Psychiatry 9th Edition: 1548.

[4] Kane JM, Stroup TS, Marder SR: Schizophrenia: Pharmacological Treatment. Kaplan \& Sadock's Comprehensive Textbook of Psychiatry 9th Edition: 1549.

[5] Swartz MS, Perkins DO, Stroup TS, Davis SM, Capuano G, Rosenheck RA, Reimherr F, McGee MF, Keefe RS, McEvoy JP et al: Effects of antipsychotic medications on psychosocial functioning in patients with chronic schizophrenia: findings from the NIMH CATIE study. Am J Psychiatry 2007, 164(3): 428-436.

[6] Ghamari Givi H, Molavi C, Heshmati R, 2010. Examine the factor structure of the scale of positive and negative symptoms of schizophrenia spectrum disorders. Journal of Clinical, Psychology. 2: 1-10.

[7] Marder SR, Hurford IM, KammenDPv: Second-Generation Antipsychotics. Kaplan \& Sadock's Comprehensive Textbook of Psychiatry 9th Edition: 3236 table: 3231.3228.

[8] Missio G, Moreno DH, Fernandes F, Bio DS, Soeiro-de-Souza MG, Rodrigues dos Santos D Jr, David DP, Costa LF, Demétrio FN, Moreno RA.. (2013). The ARIQUELI study: potentiation of quetiapine in bipolar I nonresponders with lithium versus aripiprazole.. Available:

http://www.ncbi.nlm.nih.gov/pubmed/23805994. Last accessed 28/02/2016. doi: 10.1186/1745-6215-14-190.

[9] Jin H1, Shih PA, Golshan S, Mudaliar S, Henry R, Glorioso DK, Arndt S, Kraemer HC, Jeste DV. (2013). Comparison of longer-term safety and effectiveness of 4 atypical antipsychotics in patients over age 40: a trial using equipoisestratified randomization.. Available: http://www.ncbi.nlm.nih.gov/pubmed/23218100. Last accessed 28/02/2016. doi: 10.4088/JCP.12m08001.

[10] BMC Psychiatry. 2012 Aug 2; 12: 99. doi: 10.1186/1471244X-12-99. Medication adherence and utilization in patients with schizophrenia or bipolar disorder receiving aripiprazole, quetiapine, or ziprasidone at hospitaldischarge: a retrospective cohort study. Berger A(1), Edelsberg J, Sanders KN, Alvir JM, Mychaskiw MA, Oster G. PMCID: PMC3480886, PMID: 22856540 [PubMed - indexed for MEDLINE]

[11] Stroup TS1, McEvoy JP, Ring KD, Hamer RH, LaVange LM, Swartz MS, Rosenheck RA, Perkins DO, Nussbaum AM, Lieberman JA; Schizophrenia Trials Network.. (2011). A randomized trial examining the effectiveness of switching from olanzapine, quetiapine, or risperidone to aripiprazole to reduce metabolic risk: comparison of antipsychotics for metabolic problems (C. Available: http://www.ncbi.nlm.nih.gov/pubmed/21768610. Last accessed 28/02/2016. doi: 10.1176/appi.ajp.2011.10111609.

[12] Schizophr Bull. 2012 Jun; 38 (4): 845-53. doi: 10.1093/schbul/sbq172. Epub 2011 Feb, Comparative effectiveness of second-generation antipsychotic medications in early-onset schizophrenia. Olfson M (1), Gerhard T, Huang C, Lieberman JA, Bobo WV, Crystal S. PMCID: PMC3406514PMID: 21307041 [PubMed - indexed for MEDLINE]. 